\title{
sciendo
}

Transport and Telecommunication, 2020, volume 21, no. 2, 134-148

Transport and Telecommunication Institute, Lomonosova 1, Riga, LV-1019, Latvia

DOI 10.2478/ttj-2020-0011

\section{INSTANTLY DECODABLE RAPTORQ INTERSESSIONS IN VEHICULAR ADHOC NETWORKS}

\author{
Nandhini Vineeth ${ }^{1}$, H. S. Guruprasad ${ }^{2}$ \\ ${ }^{1}$ Department of Computer Science and Engineering, \\ B.M.S. College of Engineering, Bengaluru, India \\ nandhiniv.cse@bmsce.ac.in \\ ${ }^{2}$ Department of Information Science and Engineering, \\ B.M.S. College of Engineering, Bengaluru, India \\ drhsguru@gmail.com
}

\begin{abstract}
Vehicular networks that deal with sharing of information among vehicles are gaining popularity among the automobile industry as well as the researchers. These networks are prevalent under the umbrella of Intelligent Transportation Systems (ITS) and deal with data that belongs to either the emergency category or the entertaining category. In case of emergency services, it is clear that - earlier the reception of information, lesser the commotion. The objective of this work thus has been the reduction of the end to end delay when video files are exchanged among vehicles during intersessions. The set objective is accomplished through the design and development of the technique "Instantly Decodable RaptorQ Inter-Sessions" (IDRQIS) for Vehicular Adhoc Networks and the results obtained show that this outperforms the existing popular techniques - the Network Coding and RaptorQ when applied independently to the same environment. This technique can also be applied to the upcoming unmanned vehicles.
\end{abstract}

Keywords: Vehicular Adhoc Networks, Network Coding, RaptorQ, End to End delay, Inter-session Network Coding, Instantly Decodable Network coding

\section{Introduction}

Wireless networks have opened the way for communication among devices which are positioned closer to one another as well the ones which are far apart. This can be further classified into different types like sensor networks, adhoc networks, cellular networks etc. Among these, the adhoc networks are the self-organizing networks that do not wait or depend on any infrastructure for the communication to commence and be maintained. The idea of this adhoc network has influenced researchers in trying out the technology among vehicles and the resultant, successful and very prevalent network is the Vehicular Adhoc Networks (VANETs) which falls under the umbrella of Intelligent Transportation Systems. In these networks, the vehicles are capable of playing different roles like the generators, carriers, and receivers of information that is to be shared. The communication in Vehicular networks can be between an infrastructure Road Side Unit (RSU) and a vehicle which is termed as Vehicle to Infrastructure or between two vehicles termed as Vehicle to Vehicle Communication. The information that is communicated can be either the ones like everyday news, the location of a certain prevalent place like tourist spots, restaurants etc., or it can be entertainment multimedia information like audio or movie downloads, video calls, video conference etc. The significance of these networks is exhibited when emergency messages like that of a bridge fall, accidents, traffic jams etc. are communicated which helps the vehicle reduce its contribution to the disorder prevalent in the environment of chaos (Rhoades and Conrad, 2017; MacHardy et al., 2018).

Video data that are used in any of the applications discussed above are generally huge in size. Video media opens up huge opportunities for researchers as it is very challenging to transmit these data among vehicles which are mobile. The movement of vehicles creates dynamic topology as every node change its position, direction and speed depending on its destination and sometimes environment. The various parameters that influence the successful transmission of video data are throughput, packet loss, and end to end delay among others. Though the throughput which deals with the amount of data transmitted per time unit is a widespread parameter of interest in research, the end to end delay (E-E-D) and Packet Delivery deals with the overall performance indicating the success rate of the transmission. 
The prevalent technique - RaptorQ (RQ) codes (MacKay, 2005) - which belongs to the family of erasure codes work on the forward error correction mechanism. The source data in erasure codes is divided into various symbols. The encoded symbols which are the various combinations of the source data are formed and transmitted. These symbols are received and decoded in the receiver to obtain the original data. The RQ codes are successors of the fountain codes. These fountain codes are a type of erasure codes which have the exclusive property of generating additional symbols on the fly when packet loss is encountered. The symbols exhibit a special property that any set of these symbols of the required number (even if a few are missed) if received by the receiver, makes it sufficient for the recovery of the source data. Rapid Tornado (Raptor) codes are another type of fountain codes which introduced a process before encoding- termed as a precoding process which helps in reduction of decoding delay. Some symbols are generated in addition to the source symbols and the combination of the two termed as intermediate symbols are generated and sent for encoding. These can be applied to VANETs as these suit mobile devices as the power required is low as well this technique manages the packet loss without retransmission. The RQ codes are a special stream of these raptor codes and they involve two stages of precoding- one dealing with Low-Density Parity Check (LDPC) where most of the intermediate symbols are generated and the other with High-Density Parity Check (HDPC) where the remaining intermediate symbols are generated. Encoded symbols are generated using the combination of $\mathrm{d}$ number of symbols. The value of $\mathrm{d}$ is chosen based on a probability distribution method.

Among the various techniques which could be implemented for the reduction of E-E-D, network coding (NC) (Ahlswede et al., 2000) is an interesting technique which combines the data using simple exclusive-or operation (XOR) to reduce the data that is to be transmitted. In this technique, when two nodes which are positioned far from each other i.e., one does not fall within the coverage range of the other node, are interested in communication, a third node termed as a relay node which lies in the coverage range of both these nodes, receives the data from both, mixes them up and broadcasts the mixed packet. These two nodes finally retrieve the data of the other node by applying the ex-or (XOR) operation of their own data with the received broadcasted data. This helps in reducing the total number of bytes that are to be transmitted in the transaction thus helping in the reduction of E-E-D. The encoding of NC involves the values randomly chosen from the Galois field termed as an encoding vector which is multiplied with the data in the matrix format and hence the network coding is termed as Random Linear Network Coding (RLNC).

A special extension to the network coding has been given in (Traskov et al., 2009). The advantage of this technique is the procedure of simple XOR-ing in encoding and decoding, avoiding the complexity of Galois field in encoder and reduction in decoding delay of packets in the decoder. These are applied generally in multicasting where a source transmits the same information to a group of nodes and are termed as Instantly decodable network coding (IDNC). In the first phase- packets that are not coded are multicast and the receivers give back an information regarding the receipt of packets through positive or negative acknowledgment that includes a list of packets that are not received. The two matrices are formed in each receiver, 'has set' and 'wants set'. The wants set is sent back to the source. The source now in the second phase does the combination of packets and broadcasts. In the second phase, if the source satisfies all receivers with at least one packet, then it is termed as Strict-IDNC(S-IDNC). This results in high complexity and hence Generalized IDNC is introduced which tries to consider the combination which satisfies the maximum number of receivers.

The packets received by the destination are referred using terms like Innovative packets when exactly one missing packet can be retrieved, Non-innovative packets when no new packets are available in the mixed packet and all the packets mixed up are available with the receiver and the last being Noninstantly Decodable packets or partially innovative when two or more missing source packets are seen in the combination received and hence cannot be instantly decodable.

The problem of finding the combination that maximizes the number of benefitted users is formulated as Integer Linear Program (ILP) and proven to be NP-Hard. This is termed as weighted sum S-IDNC (Sorour and Valaee, 2015).

In this work-IDRQIS, the combination of the techniques like RLNC, IDNC, and RaptorQ codes are used in InterSessions (IS) where two vehicles have data that need to be exchanged among themselves.

This work suits the unicast environments where two vehicular nodes that are not in the range of each other want to exchange files among themselves. In real-world any number of parallel transmission are expected to happen and hence as a simulation, two simultaneous transmissions have been implemented in this work to observe the challenges that are faced during the process. This can hence suit environment like skype communication but with a difference that the communicating parties are traveling in their vehicles. 


\section{Materials and methods}

An extensive survey of the existing literature in the field of network coding, RaptorQ codes, end to end delay measurement and video data transmitted after being encoded by the two above two techniques is done and presented in this section.

\subsection{Network Coding}

Network Coding combines original packets and transmits the combined packet towards the destination via relay nodes. Random Linear Network Coding (RLNC) is the type of NC applied generally in many applications, but there are various methods in literature proving that RLNC does not give a better performance with Galois field (GF-2). This works better with large Galois field but with huge complexity. This literature shows that if uncoded packets are transmitted prior to the transmission of coded packets via two relay nodes, performance is improved. The number of packets sent to these two relay nodes is decided based on the probability calculation of the link duration etc. (Monteiro et al., 2017). There are various categories of network coding like generation based, intersession, intrasession etc. The generation based is the category where the message to be transmitted is divided into blocks which are in turn separated into generations and the mixing of the data that belongs to the same generation is done, intersession $\mathrm{NC}$ is applied when two nodes need to exchange data among themselves, both these nodes transmit their data to a recoder which mixes the data and broadcasts, intrasession NC is where data from the same flow is being mixed (Vineeth and Guruprasad, 2014).

The video has been a significant parameter of interest for many researchers. Markov Decision Processes (MDP) has been used by researchers for the transmission of video contents. When a finite transmission horizon is seen, MDP has been employed to minimize the distortion of video streams with an optimal NC selection policy. The computation of these optimal policies is made intractable with the help of action spaces and the dimensionality of MDP's state. This computational complexity is reduced using a simulation-based dynamic programming algorithm (Nguyen et al., 2007; Nguyen and Nguyen, 2009).

Dynamic programming is considered in (Skevakis and Lambadaris, 2016) for improving the file transfer completion time. A Markov decision process is modeled which handles the computational complexity and storage in the receiver through RLNC. Broadcast networks exhibit a good throughput and less transmission delay.

Feedback is another approach where the performance of the network can be enhanced. This is the technique used in (Yu et al., 2017). The data is transmitted in both coded as well as an uncoded form through two phases. In the second phase, packets belonging to the same generation are grouped and encoded. There is a feedback required to be sent by the intermediate nodes to the source regarding the set of packets received in the receiver nodes. Throughput and decoding delay are the parameters of interest. Generations are further divided into sub generations and the packets belonging to the same generation are combined linearly. This is shown to be an NP-complete problem. The technique NC with cooperative data exchange is used that helps the packets reach the destination within deadlines.

Another work that shows the problem as NP-Hard is (Sui et al., 2016) where Integer programming approach is used. The general problem is solved by using the auxiliary graph model.

\subsection{Instantly Decodable Network Coding}

Instantly decodable network coding is an interesting stream of network coding which orients mostly towards the reduction of delay.

Time critical applications are a significant area of research. The nodes are made to simultaneously communicate with the base station as well with the other devices through two interfaces. Special care is taken for in-order delivery of packets by authors during implementation. IDNC is put into work for addressing the dual delivery delay problem which is equivalent to an independent maximum weight set selection problem. The authors compare the mean decoding delay (DD) and completion time (CT) of various algorithms like cellular IDNC, D2D IDNC among others for a network composed of various devices. They show their proposed algorithm exhibit the least mean CT and DD (Karim et al., 2017).

Delay minimization while transmitting the data over Markovian ON-OFF channels is done using instantly decodable network coding. Due to the reason that Strict IDNC requires a new solution to every node in every transmission, it is shown to be NP-hard by the researchers (Drinea et al., 2009).

The concepts of Strict IDNC and Generalized IDNC are both used in this technique. The authors use G-IDNC only conditionally. Un-coded packets transmitted in the first stage then coded packets are transmitted after getting a feedback of missing packets from the set of receivers. Various observations 
have been presented here like proving that it turns out to be NP-Hard when the average packet decoding delay of S-IDNC is to be minimized, the graphs of both share the same chromatic numbers. The design of the coding algorithm of the source has been made based on the feedback given by the receiver or the intermittent nodes (Yu et al., 2015).

Maximizing the density of coding opportunities by maximizing the number of innovative packets in IDNC is done by (Sorour and Valaee, 2012; Sorour and Valaee, 2013). In the other work (Sorour and Valaee, 2010) the minimization of the mean completion delay in wireless multicast has been targeted by formulating a stochastic shortest path (SSP).

When video data is transmitted, layers of video frames are considered for processing. IDNC is used in the transmission of such video frames. Assigning different priorities to different layered video streams and transmitting them based on the priority is a strategy used to make the receivers get the minimum required frames before the expiry of the deadline. A threshold is derived for the same reason which deals with the minimum number of packets that need to be received by all receivers to obtain the most important layers of video data (Karim et al., 2016).

Considering the parameters decoding delay and completion time, a relationship between the two has been considered by the authors recently. It has been seen that finding the optimal overall completion time is NP-Hard. Completion time is reformulated as an online optimization problem involving the decoding delay experienced in feedback termed as imperfect feedback. The main objective of the algorithm is to reduce the probability of an increase in the maximum decoding delay experienced which reflects on the completion time experienced. The online optimization problem is solved by two greedy heuristics. The authors claim that they are able to achieve a lower mean completion time compared to the ones available in the literature (Ambadi et al., 2017; Douik et al., 2017a). IDNC is also used in multicasting such video data to different viewers in some work as the video is received and viewed on the basis of their requirements (Greco et al., 2016).

The device to device communications has been done in two phases in (Douik et al., 2016) where the combination of GF-2 and IDNC applied with receiver's feedback is used. During the first phase, the base station transmits data to the end nodes when the nodes send back an acknowledgment of the packets received. During the recovery phase, the receiver nodes try sharing the combination of non-instantly decodable packets among themselves. An algorithm is proposed which will select the optimal node which will transmit the best combination which could benefit the maximum number of nodes.

The optimal utilization of spectrum used by the base stations in $5 \mathrm{G}$ networks is set as the objective in (Douik et al., 2017b) to increase the data rate. Simple XOR-ing replaces the complicated operations over Galois field during encoding and hence the complex matrix inversion in decoder is also avoided. When multicasting is done, the methodology followed to combine the packets satisfies maximum users. Index-coding aims at the reduction of transmission time for erasure free channels. Reaching the global optimal of index coding problem is NP-Hard. Greedy algorithms are proposed to solve index coding problems.

As unicasts will be more prevalent in point to multipoint networks, multiple unicasts have been tried out in (Hai et al., 2016). The general solution is achieved through a framework involving the bin packing problem. Minimum completion delay is the objective. An efficient greedy algorithm is used to achieve a low complexity. The number of retransmissions is proved to be reduced.

A cross-layer scheme that incorporates different channel rates of various users in the decision process of both the transmitter message combination and the rate with which they are transmitted is proposed in (Douik et al., 2017c). Asymmetric rates for receivers reflect a new coding combination for various receivers and broadcasts rates. The completion time minimization problem is shown to be intractable and is solved as a maximum weight clique problem over a newly designed rate-aware IDNC graph. Simulation results show that this cross-layer design largely outperforms the un-coded transmission strategies and classical IDNC Schemes.

There are various supporting streams like routing, mobility etc., for the efficient transmission of the data in these networks. The various routing protocols like Adhoc on Demand Routing Protocol (AODV), Destination Sequenced Distance Vector (DSDV) and Open Link State Routing Protocol (OLSR) are applied in VANETs and the various delay parameters are measured in (Vineeth and Guruprasad, 2015a) when Network coding is used for encoding and decoding. The results show that AODV which is a reactive protocol that best suits VANET environment but shows more delay compared to the proactive protocols DSDV and OLSR which opens a challenge for the reduction of delay. As the vehicles are mobile, a mobility model needs to be set to bring in their actual behaviour. The various mobility models like constant position mobility model, constant velocity mobility model etc. are applied 
on the VANET environment where the network coding is applied and their influence on the delay parameters are measured (Vineeth and Guruprasad, 2015b).

\subsection{Delay aware fountain codes}

The video, being the media of interest and delay that influences the performance of video transmission being the significant parameter of IDRQIS algorithm, the survey orients towards video and delay and the various works in existing literature on these. The efficiency of any work done with the objective of achieving a lesser delay would not be complete if it exhibits a considerable packet loss. The packet delivery ratio is a significant parameter which needs be observed with the delay parameter in many scenarios. A technique that helps in an efficient transmission in an environment where packet loss is more is the RaptorQ technique, a special category of fountain codes.

The video coding and channel coding tasks are put together to improve the quality of video streaming in (Mammeri et al., 2016). Two protocols- one that deals with the fountains which are delay aware and the other which is the low complex version in which the computational complexity is reduced are introduced. The techniques involved use an adaptively selected sliding window and a sampling pattern chosen on a video rate. Real-time test beds are also used for testing the algorithms in many experiments. One such testing for comparing Erasure Coding-RTP and RTP is done in (Heo et al., 2015) where two tablets are used in place of On-Board Units and Wi-Fi is used in place of 802.11p and MPEG files are transmitted. Every unit of data is processed by the MPEG media transport layer and further subdivided into functional units. Every frame is decoded by ffmpeg decoder to work on the functional unit instead of the full processing units. The functional units are reassembled to obtain the processing unit. Redundant data is introduced by XOR in methods where erasure coding is prevalent but the packet loss is simulated through burst error model here. The same speed and distance in between the vehicles carrying the tablets have been maintained and have tested for E-E-D, usage of bandwidth etc. It is proved that the packet loss is considerably reduced in EC-RTP but the bandwidth and delay are better seen in RTP which makes this an open area for research.

RFC6330 (Shokrollahi et al., 2011) is the document that provides the various header formats, complete details of encoding and decoding of RaptorQ codes.

The performance of RaptorQ codes is explored to be improved through various techniques. One such technique is (Vineeth and Guruprasad, 2018) where the encoding and decoding are done using threads. The main video file to be transmitted is divided into three and supplied to three threads which encode them in parallel. Once encoded, these three encoded pieces are concatenated and the resultant data is made into packets for transmission. Once these are received in the recoder, these are again divided into three sets of data which are supplied again to three threads which decode the data in parallel. The objective is the reduction of E-E-D is achieved by reducing the encoding and decoding delay with the introduction to parallelism.

Fountain codes have proven to show good results in multimedia transmission. Rateless coding depends on the channel and the video coding and is delay aware. Internet of things can also be applied to these two types of coding. The model predictive control and fountain codes introduced with delay aware sliding window in (Sun et al., 2016a) have resulted in an optimum decoding ratio that outperforms many video communication algorithms. The extension of this is the dynamic selection (Sun et al., 2016b) of window size. As relay nodes play a very important role in multimedia transmissions, the research on relay nodes has gained popularity.

Considering the performances of the fountain-based system and relay-based systems in consideration with transmission delay, (Asareh and Fujii, 2013) has shown that fountain-based outperforms the relay-based. The significant part played of the message size, packet size and signal to noise ratio in relay-based systems are measured. Another work which orients towards relay node selection is (Gul et al., 2016) which involves time sharing, suggests the best relay node in multimedia transmissions. The results dealing with decoding overhead and the probability of failure prove that this technique is better compared to amplify and forward schemes. Considering the success probability of random linear FC, the redundancy of data is analyzed to be high which is reduced in (Qureshi, 2016). The codeword is generated based on the linear combination of code words but it is taken care not to include the set of code words used earlier for predefined iterations. This method has shown a significant reduction in the number of transmissions for multicast networks.

Another work on unicasting and multicasting in VANETs involving fountain codes is done in (Zhang et al., 2016) where stationary vehicles with surplus storage and energy act as charging stations for the moving vehicles with limited storage and energy. The mobile vehicles act as relay nodes in between 
the stationary vehicles. The work shows that to achieve a minimum delay with multicasting, a unique coding rate is to be used. A greedy algorithm is used for this rate selection. It is observed that the delay increases when the number of redundant packets exceeds a particular limit.

With the objective of reduced complexity and delay, (Hayajneh and Yousefi, 2015) works on generations which are subsets of the file to be transmitted. The highest priority contents are kept in the first partition and an overlap of generations are seen. The last partition of the nth generation overlaps with the first partition of the $n+1^{\text {th }}$ generation. These, when applied on heterogeneous channels, outperform LT codes.

The other factors that influence the delay can be the size of the data that is being transmitted and the size of the packets which is used to transmit the data.

As the packets of the same size show better efficiency, packets of unequal sizes are padded with zeros which in turn results in increased overhead. This challenge for the performance of the network is dealt with in (Taghouti et al., 2016) where zero padding is avoided where symbols are processed in an appropriate finite field. The trade-off between the complexity and the overhead is managed by using the on the fly decoding at the macro symbol level.

When the techniques prove to be better with respect to parameters of interest, it would be advantageous to analyze both together showing a comparison. i.e. according to literature, NC and RQ have shown better results with respect to the E-E-D parameter.

Network Coding and RaptorQ codes are applied and the E-E-D is measured by varying the file size and the packet size, the results measured are compared. It has been observed that when the file size is smaller, RaptorQ codes play a better role while larger files can be transmitted by applying the Network Coding technique (Vineeth and Guruprasad, 2017a). The other work that works on these two techniques is (Vineeth and Guruprasad, 2017b) where the better of the two techniques is suggested that best suits the environment given the various parameters like the nodes interested in communication, the file size and the packet size. The packet delivery ratio which is a supportive parameter to the E-E-D is another parameter of interest here which is just measured and reported and not worked upon. Hence both the parameters are given importance in this proposed work.

\section{Proposed work}

The main objective of this work- IDRQIS is to reduce the E-E-D encountered in scenarios where intersessions are seen. Intersessions are said to be encountered in environments when two vehicles have data to be exchanged among them. This may suit mobile conferences, safety message exchanges, and entertainment data which are generally disseminated among vehicles in VANETs. The main parameter of consideration in this work is E-E-D. When experiments show a lesser E-E-D but are observed with a significant packet loss, the efficiency of the network is reduced considerably and time-sensitive data like video data cannot be played efficiently before their deadline. Hence in this work, both the parameters are taken care. When the percentage of packets lost is greater than $10 \%$ of transmission i.e. when the packet delivery ratio is less than $90 \%$, then the lost packets are retrieved with the help of another node termed as helper node in this work. The two techniques-Network coding and RaptorQ codes are applied to the environment. A special category of Network coding termed as Inter-Session Network Coding (ISNC) is considered for experimenting the E-E-D reduction of the video file transmissions. Another special stream of network coding termed as Instantly Decodable Network Coding (IDNC) and RaptorQ (RQ) codes support the way packet losses are managed. The usage of the combined advantages of the above three techniques when video data is transmitted in the WAVE environment has a significant role in the achievement of the set objectives.

The VANET environment is set up using WAVE 802.11p standards in Network Simulator 3. Thirty-two VANET nodes are created which are placed in the form of a grid of size $4 \mathrm{X} 8$. The number of nodes does not make much significance except they complete a grid formation. They have applied mobility with the first 16 nodes set with a speed of $40 \mathrm{Km} / \mathrm{Hr}$ and the next 16 set with $80 \mathrm{Km} / \mathrm{Hr}$. AODV, the reactive Protocol is applied in the environment that helps in establishing the routes among the vehicles and the mobility model is set to Constant Velocity mobility model that exhibits different but constant velocity to the environment. One of the three different files of varying sizes- $10 \mathrm{~KB}, 20 \mathrm{~KB}$ and $38 \mathrm{~KB}$ are taken as inputs.

According to the results observed in (Douik et al., 2017c), Network coding suits environment where the file to be transmitted is huge and packet size is small and RaptorQ suits environment where file size is less and packet size is bigger say 1024. Hence this work deals with combining the advantages of both and brings in a technique which works better in all environments. 


\subsection{Proposed algorithm}

\subsubsection{Algorithm:}

1. The RLNC is applied on the packets by the two nodes of interest and the resultant encoded data is transmitted to the recoder. The recoding procedure decodes, mixes the packets and again encodes them to be made ready for transmission. The E-E-D is calculated as discussed in step 12.

2. A similar wave environment is set up and the RaptorQ technique is applied to the same two nodes of interest used for communication considered above. The E-E-D is calculated as discussed in step 12.

3. In the implementation of IDRQIS, the number of hops between these two nodes of interest is measured by sending a dummy packet from each of the nodes.

4. Case 1:

If the number of hops between the nodes is one, i.e. single hop nodes, then each node creates packets containing the contents of the file and transmits them to the other node. The data transmitted here is the raw uncoded data. No Encoding and decoding procedures are applied here. Every packet received is immediately decoded.

5. Case 2:

If the number of hops between the two nodes is two,

- A node which is at one hop from each of these source nodes, i.e. a common node which is in the range of both the nodes which acts as the recoder node is selected.

- Each node creates packets containing the contents of the file to be transmitted to the other node and transmits them to the recoder. The recoder node mixes both the packets received and broadcasts the mixed data. The data transmitted here is the raw uncoded data. No Encoding and decoding procedures are applied here. Every packet, when received at the destination, is decoded and mixed with the packet contents generated from its own file. This retrieves the contents of the file it is awaiting.

6. Case 3:

If the number of hops is more than two, indicating that there are multiple hops in between the two nodes, the following steps are followed.

- A node which is located between the two source nodes is selected which acts as the recoder node.

- One helper node for each of the source nodes which is closer to the recoder is selected.

- Each source encodes the contents of the video data to be transmitted using the random linear network coding procedure, generate packets and transmits them towards the recoder.

- The recoder node receives two transmissions from two source nodes. It decodes them using random linear network coding procedure to generate the actual contents of video data present in the packets from both the source nodes. Inter-session network coding is achieved when the recoder mixes the contents of these packets that are created by the nodes and destined for the other peer. In Intersessions, when two nodes say A and B are involved, the node $\mathrm{A}$ which acts as a source in transmission 1 acts as a destination in transmission 2 and the node B which acts the destination in transmission 1 acts as the source in transmission 2 . The recoder encodes these mixed packets using RaptorQ encoding technique and transmits them towards all the four nodes- two destination nodes and two helper nodes.

7. If the transceiver node receives a packet,

- it is immediately RaptorQ decoded.

- These decoded packet contents are mixed with the contents of its own video file so that the packet contents of the other file is retrieved.

- The wait for the next packet starts.

8. If the packet is lost, then a new thread is created which sends the request for the missed packet to the helper node whereas the main thread continues to receive packets. The helper node retrieves the packet contents, transmits the data packet towards the requesting node, which follows the procedure of decoding and mixing to retrieve the contents transmitted by its peer node.

9. The E-E-D for all three cases is calculated as discussed in step 12.

10. The packet sizes are varied as 256,512 and 1024 bytes during the application of the above three techniques and the results are observed for comparison and plotted.

11. The file sizes are varied as $10 \mathrm{~KB}, 20 \mathrm{~KB}$, and $38 \mathrm{~KB}$ and the results are observed for comparison and plotted. 
12. The end to end delay is calculated in this work as the time difference between the decoding of the last packet and the raw data being filled in the buffer for encoding. The E-E-D is calculated for the two transmissions and the greater value of the two is considered as the outcome value that is used for comparison with the other techniques.

\subsubsection{Graphical representation:}

Node A, Node B- Encode the video data using Network Coding and transmit to recoder

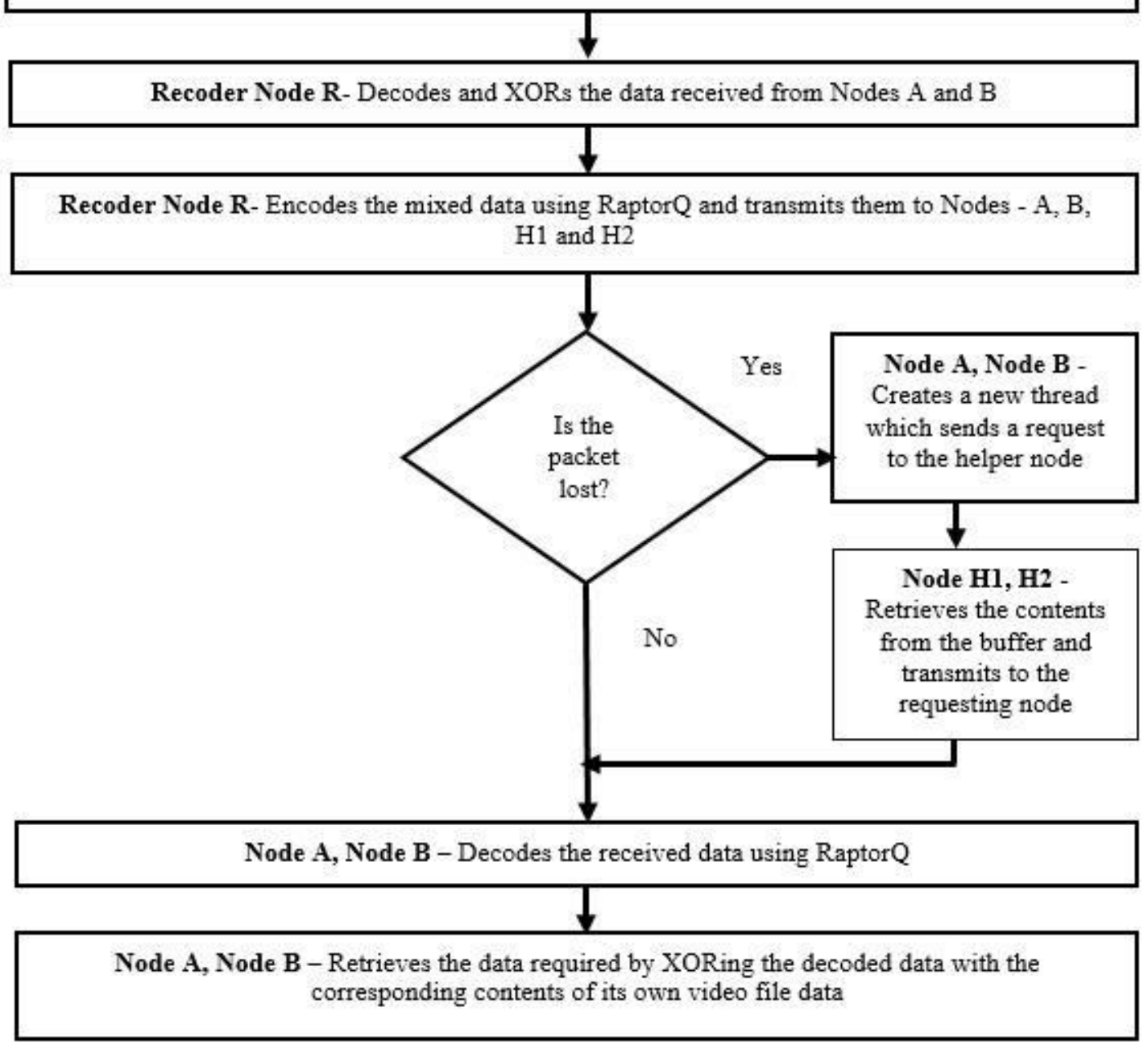

Figure 1. Graphical Representation of IDRQIS

\subsection{Proposed architecture}

\subsubsection{Case 1:}

\begin{tabular}{|c|c|}
\hline Node A & Node B \\
\hline $\begin{array}{lrr}\text { The uncoded video } \\
\text { data } & \text { VD1 } & \text { is } \\
\text { transmitted to Node B }\end{array}$ & $\begin{array}{lrr}\text { The } & \text { uncoded video } \\
\text { data } & \text { VD2 } & \text { is } \\
\text { transmitted to Node } A\end{array}$ \\
\hline
\end{tabular}

Figure 2. Single hop Communication between two nodes A and B in IDRQIS

Figure 2 shows graphically the procedure of communication which the two nodes which are at single hop distance from each other follow. Here the raw uncoded data is transmitted to the other node. 


\subsubsection{Case 2:}

The Figure 3 shows graphically how two nodes which are at two hops to each other communicate. The uncoded video is transmitted to a recoder by the two nodes. The recoder node $\mathrm{R}$ receives the uncoded data from both the nodes $\mathrm{A}$ and B. They mix the two packets received and broadcasts them. The broadcasted data is received by the nodes $\mathrm{A}$ and $\mathrm{B}$, which mix the received packet with its own corresponding data from the file.

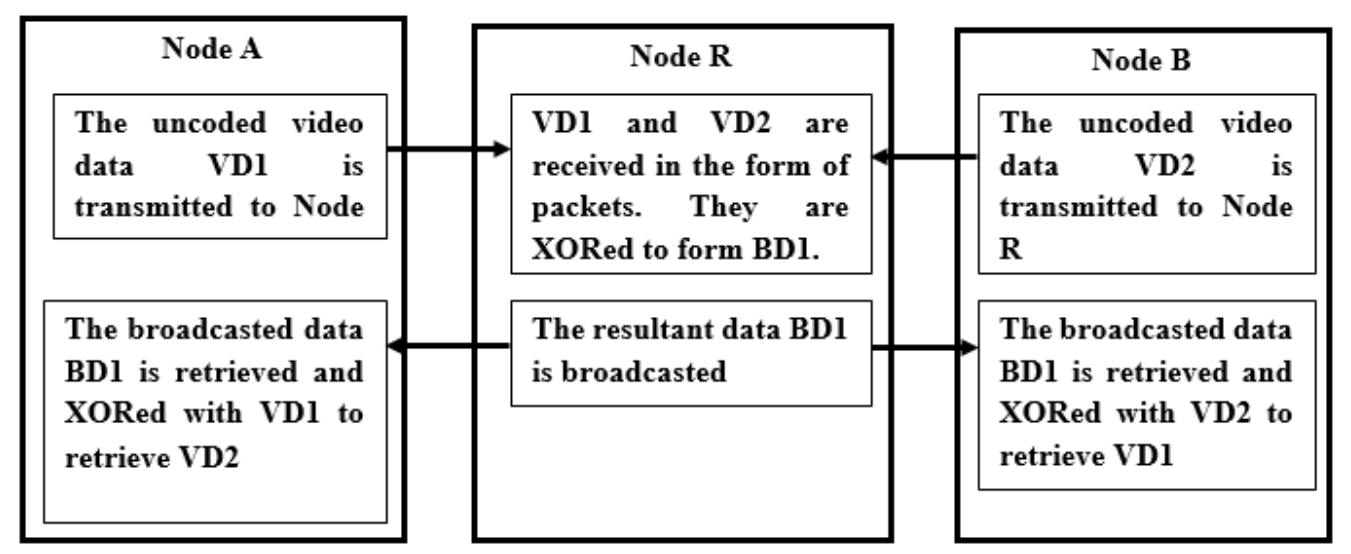

Figure 3. Two hop Communication between two nodes A and B in IDRQIS

\subsubsection{Case 3:}

The operations of the technique IDRQIS is shown in Figure 4. The detailed working of this case is shown in the proposed algorithm.

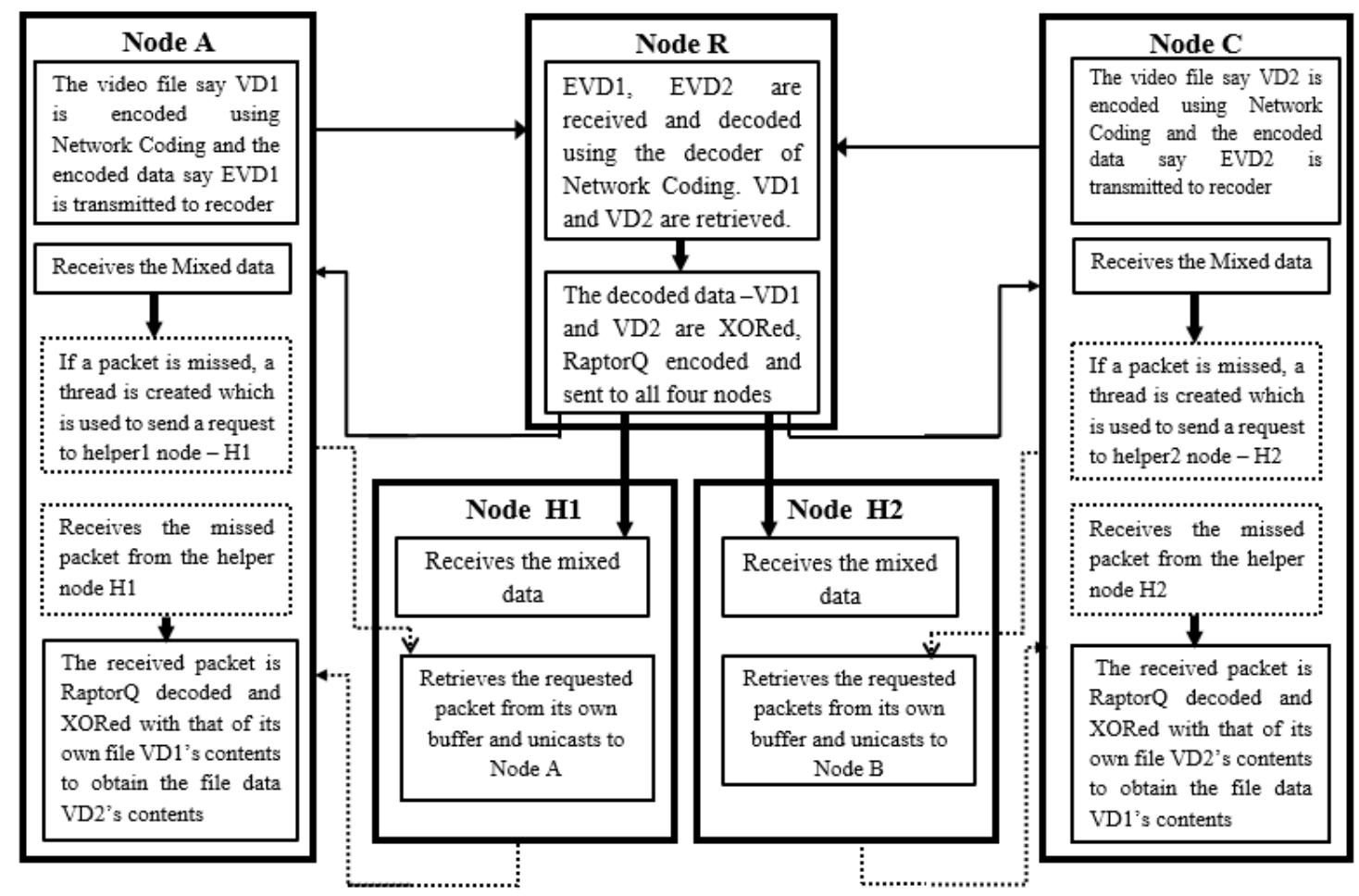

Figure 4. Multi-hop Communication between two nodes A and B in IDRQIS

\section{Results and discussion}

The simulator used for the implementation of the above algorithm is Network Simulator 3 and some of the important modules used are a wave, aodv, IPV4, MobilityModel etc. The thirty-two vehicles 
created for the application of the scenarios are applied to the wave environment under the standard 802.11p. The numbers can be increased or decreased according to the scaling required. The implementation becomes easier in simulators when the number of vehicles to be included becomes more, compared to the real-time testbeds and hence adapted in this work.

The reactive protocol AODV which makes the dynamic decisions has been selected as the mobile vehicles result in dynamic topology. All the three techniques are applied with three packet sizes 256 bytes, 512 bytes and 1024 bytes where each environment is fed with three different files sizes of 10KB, $20 \mathrm{~KB}$, and $38 \mathrm{~KB}$. The inputs - source, destination, recoder, helper1, helper2, packet size, and file size are accepted dynamically. The source and destination nodes accepted are for one session and their roles are reversed for the second session. The various parameters and the values initialized in the experimentation is tabulated in Table 1 which is included in the last section.

The network coding technique is applied for the encoding and the decoding procedures in the environment created and the E-E-D for the completion of each session is noted. The maximum of the two is considered for comparison. Similarly, the RQ encoding and decoding are done and the E-E-D is observed using the same procedure as NC.

The new technique - Instantly Decodable RaptorQ Inter-Sessions (IDRQIS) in VANETs is also applied and the E-E-D is measured. According to the new technique in multi-hop, the two transmissions are done by two source nodes say A and B towards the Recoder say R. The recoder decodes the received packets, mixes the packets and sends them to the receiver nodes and the helper nodes. The complete time taken for each of the transmissions are observed, the higher value of the two is accepted as the finalized E-E-D.

The supporting parameter in this work is the Packet Delivery Ratio (PDR). When the E-E-D is measured to be small, and the PDR is also measured to be less than ninety percentage, the transmission is not considered successful as the video data required for playback cannot be retrieved. Hence PDR becomes a significant parameter influencing the efficiency of transmissions. The E-E-D is the difference between the completion of decoding in the destination and the time the video data is read. The PDR is measured using the flow monitor module of NS3. In all techniques, the average PDR of the two transmissions is considered.

The module Gnuplot in NS3 is used for the generation of graphs. The varying combinations of the three single hop scenarios, three two hops scenarios and six multi-hop scenarios with varying file sizes and packet sizes are considered for the observations. The results presented here are the average of the EE-D and PDR values measured for all three cases of single hop intersessions, the average of the E-E-D and PDR values measured for all three cases of two hops intersessions and the average of the E-E-D and PDR values measured for all six cases of multi-hop intersessions. The plotted graphs are included from Figure 5 to Figure 13.

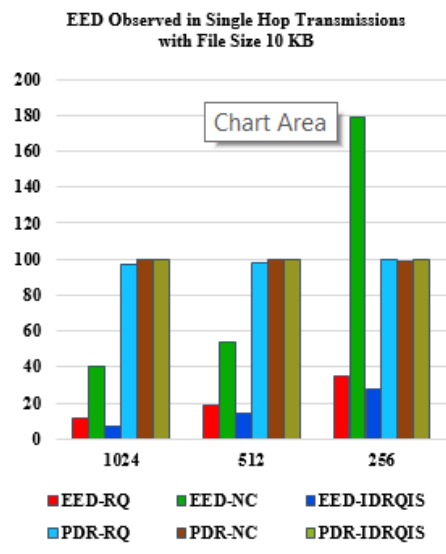

Figure 5. E-E-D and PDR Observed in Single Hop Transmissions with File size $10 \mathrm{~KB}$
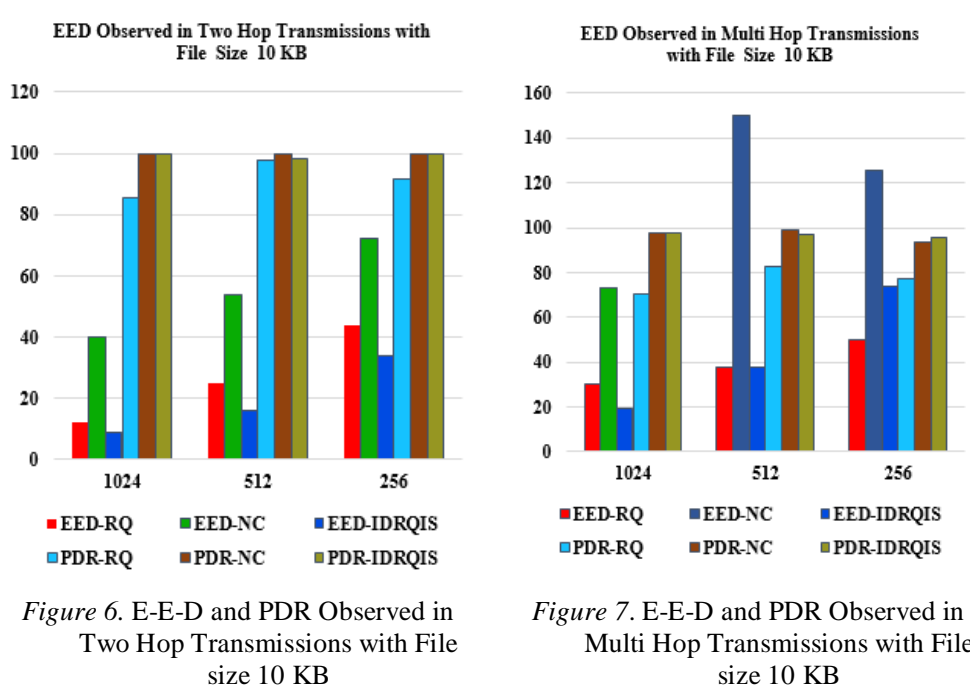

Figure 7. E-E-D and PDR Observed in Multi Hop Transmissions with File size $10 \mathrm{~KB}$

\subsection{Single hop nodes with file size- $10 \mathrm{~KB}$}

Considering the E-E-D measured in the three cases of varying packet sizes-(i) 256 bytes, (ii)512 bytes and (iii) 1024 bytes as shown in Figure 5, when the source and destination nodes are at one hop from each other, the result shows that IDRQIS algorithm outperforms the RQ and NC technique. The reason is 
that in IDRQIS algorithm the raw data is transmitted as such and no encoding and decoding is done whereas in the other two the encoded data is transmitted and the received data is decoded which results in more delay. i.e., No encoding and decoding delay is encountered in the communicating nodes in IDRQIS technique. The PDRs measured in all three cases as shown to be greater than $90 \%$.

\subsection{Two hop nodes with file size-10KB}

Considering the E-E-D measured in the three cases of varying packet sizes-256 bytes, 512 bytes, and 1024 bytes as shown in Figure 6, when the source and destination nodes are at two hops from each other, the result shows that our algorithm outperforms the RQ and NC technique. The reason is that in addition to the absence of encoding and decoding delay mentioned above in IDRQIS algorithm, an intermediate recoder node which is in between both the nodes is chosen and hence the raw data does not travel till the destination whereas in the other two the encoded data is transmitted towards the destination and the received data is decoded. In spite of a little processing delay being introduced by the recoder node for mixing, the final EED Values observed here is less compared to the other two which involves the encoding and decoding procedures. The PDRs measured in all cases as shown to be greater than $90 \%$ except RQ exhibiting a lesser PDR when the packet size is set as 1024 bytes.

\subsection{Multi-hop nodes with file size-10KB}

When the number of hops is more than two, with packet size 1024 bytes as shown in Figure 7 , the E-E-D measured is seen to be less compared to RQ and NC. With the packet sizes being 512 and 256 bytes, the EED measured by RQ is seen to be less than IDRQIS. When the PDR of RQ is seen, both the cases exhibit the measured ratio to be less than $90 \%$ which is considered as the minimum cut-off data to be received for successfully completing the transactions. The techniques IDRQIS and NC measure a packet delivery value greater than $90 \%$.
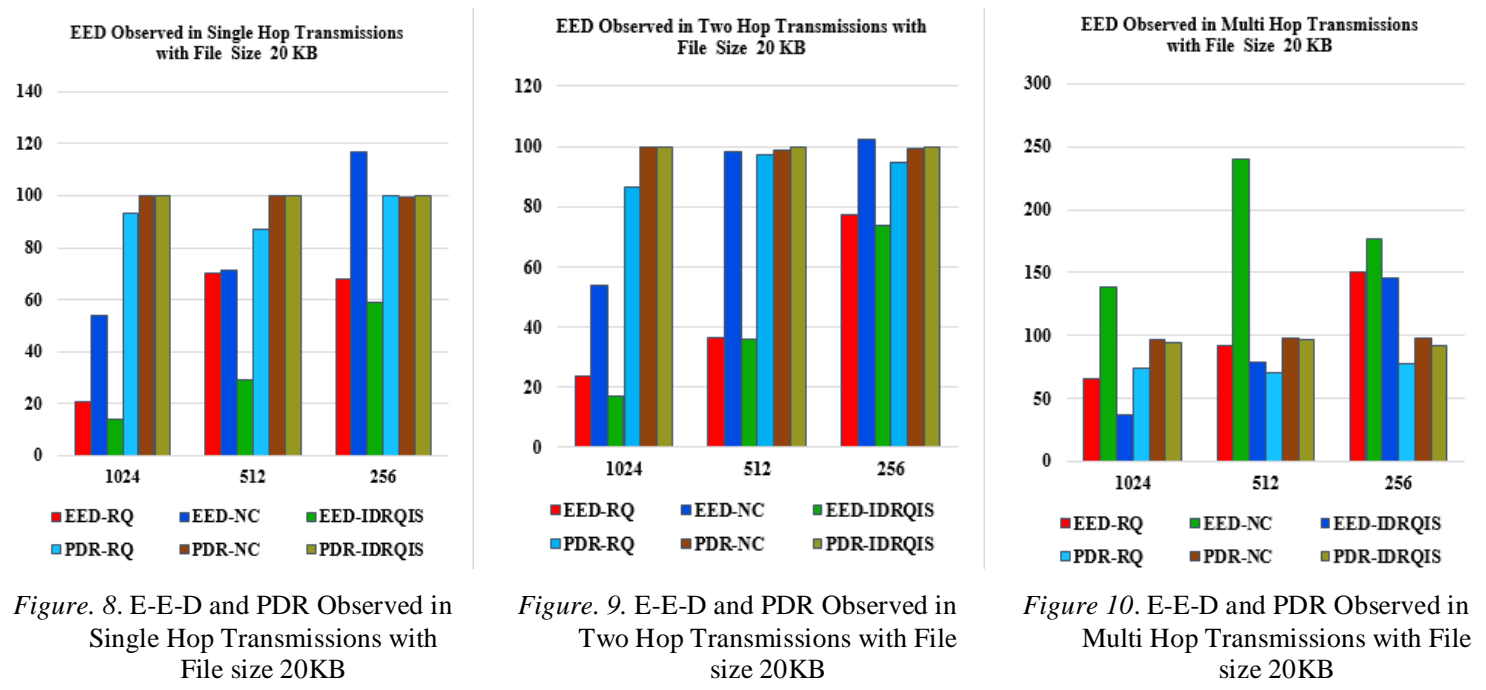

\subsection{Single hop nodes with file size-20KB}

Considering the E-E-D measured in the three cases of varying packet sizes-256 bytes, 512 bytes, and 1024 bytes as shown in Figure 8, when the source and destination nodes are at one hop from each other shows that our algorithm outperforms the RQ and NC technique. The reason is that in IDRQIS algorithm the raw data is transmitted as such and no encoding and decoding is done whereas in the other two the encoded data is transmitted and the received data is decoded which results in more delay. The techniques IDRQIS and NC measure a packet delivery value greater than $90 \%$ whereas the RQ fails only when the packet size is 512 bytes.

\subsection{Two hop nodes with file size-20KB}

The IDRQIS algorithm exhibits a lesser E-E-D measured in all three cases bytes as shown in Figure 9 compared to the RQ and NC techniques. The reason is that of the absence of encoding and 
decoding techniques as compared to the RQ and NC techniques. The PDRs observed in all cases of the three techniques are above $90 \%$.

\subsection{Multi-hop nodes with file size-20KB}

The IDRQIS algorithm exhibits a lesser E-E-D measured in all three cases bytes as shown in Figure 10 compared to the RQ and NC techniques. The reason is that of the presence of the intermediate node which receives the data in full and processes it according to the procedures discussed above. The propagation delay is reduced here compared to RQ and $\mathrm{NC}$ with an introduction to the processing delay. The techniques IDRQIS and NC measure a packet delivery value greater than $90 \%$ whereas the RQ fails in all three cases.

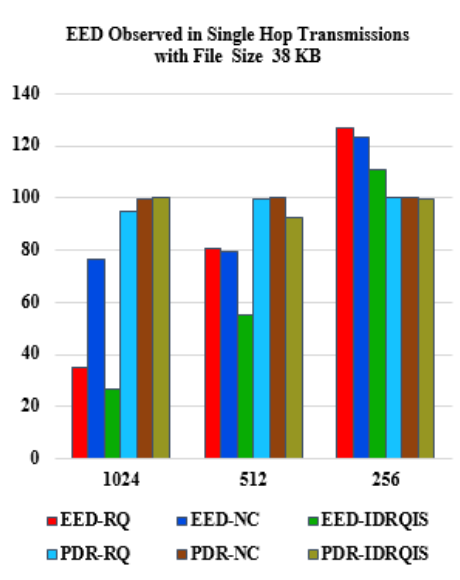

Figure 11. E-E-D and PDR Observed in Single Hop Transmissions with File size $38 \mathrm{~KB}$

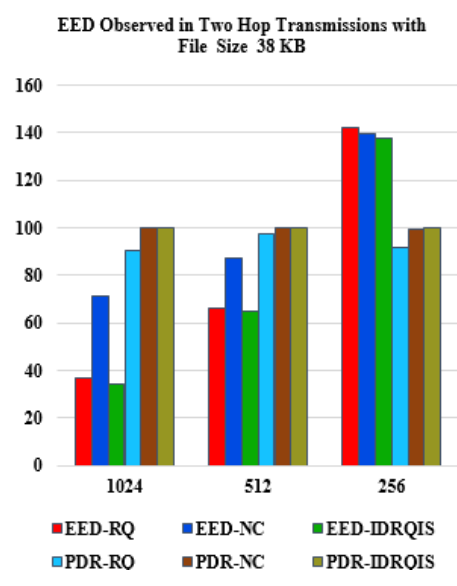

Figure 12. E-E-D and PDR Observed in Two Hop Transmissions with File size $38 \mathrm{~KB}$

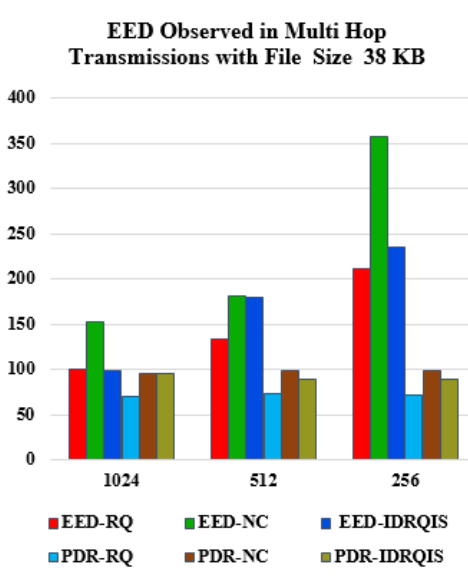

Figure 13. E-E-D and PDR Observed in Multi Hop Transmissions with File size $38 \mathrm{~KB}$

\subsection{Single hop nodes with file size- $38 \mathrm{~KB}$}

The EED measured proves the technique IDRQIS better than the other two bytes as shown in Figure 11. The reason being the raw uncoded data transmission of IDRQIS compared to the encoding and decoding done with RQ and NC. The PDRs observed show successful completion by all three techniques.

\subsection{Two hop nodes with file size-38KB}

The IDRQIS algorithm exhibits a lesser E-E-D measured by in all three cases here compared to the RQ and NC techniques bytes as shown in Figure 12. The reason is that of the absence of encoding and decoding techniques as compared to the RQ and NC techniques. The PDRs observed in all cases of the three techniques are above $90 \%$.

\subsection{Multi-hop nodes with file size-38KB}

When the number of hops is more than two, with packet size 1024 bytes, the E-E-D measured is seen to be less compared to RQ and NC bytes as shown in Figure 13. With the packet sizes being 512 and 256 bytes, the EED measured by RQ is seen to be less than IDRQIS. When the PDR of RQ technique is looked into, both these cases exhibit the measured ratio to be less than $90 \%$ which is considered as the minimum cut-off data to be received for successfully completing the transactions. The techniques IDRQIS and NC measure a packet delivery value greater than $90 \%$.

Thus the IDRQIS technique designed achieves a lesser E-E-D compared to the two prevalent techniques Network Coding and RaptorQ techniques. The above parameter is also supported by the parameter Packet Delivery Ratio which supports the results observed with respect to E-E-D.

\section{Conclusion}

In vehicular networks, the transmission of video data is very challenging and hence very prevalent in research. As the end to end, delay is a critical parameter when the video data is considered, this work has the objective to reduce the end to end delay experienced. As the number of vehicles can be made 
scalable during experimentation, simulation tools are considered. The packet delivery ratio is identified as a supporting parameter, as a lesser E-E-D measured with lesser PDR shows the inefficiency of the technique. It is also taken care that the packet delivery ratio is maintained above $90 \%$ which makes the end to end delay observed and presented a significant and useful one. The intersessions are created using intersession network coding (ISNC) technique, helper node concept and the instantly decoding concept are adapted from the instantly decodable network coding (IDNC) and an efficient packet delivery is supported by the RaptorQ codes (RQ). The usage of threads introduces parallelism in the encoding and decoding phases of the RaptorQ technique as well during seeking the missed packet from the helper nodes. In this research work, an efficient architecture for vehicular networks with an appropriate combination of the routing protocols, mobility models, encoding and decoding techniques, vehicle density, file size, packet size etc., has been built and tested using Network Simulator-3. The results show the end to end delay experience by the hybrid technique - IDRQIS is less compared to the two prevalent techniques - Network Coding and RaptorQ techniques when applied independently. The parameter packet delivery ratio which plays a significant supporting role for the end to end delay parameter is also maintained above $90 \%$ thus enhancing the efficiency of video stream transmissions in Vehicular Networks. As all the PDRs seen in the forward direction exhibited values above $90 \%$, no action has been taken on packet drops in the forward transmission. In the reverse transmission i.e. from the recoder to the destination (last mile), the inefficiency of transmission that would have been caused due to the packet loss is overcome by the use of helper nodes. The work orients towards unicast communication. This can be applied to multicast communication in future. This can be used in an emergency environment of an accident where the video data of the patients can be transmitted to doctors in hospitals who in turn can advise the caretakers regarding the first aid, skype communications as well in unmanned vehicles.

\section{References}

1. Mammeri, A., Boukerche, A., Fang, Z. (2016) Video Streaming Over Vehicular Ad Hoc Networks Using Erasure Coding. IEEE Systems Journal, 10 (2), 785-796. DOI: 10.1109/ JSYST.2015.2455813.

2. Ahlswede, R., Cai, N., Li, S. R., Yeung, R. W. (2000) Network Information Flow. IEEE Transactions on Information Theory, 46 (4), 1204 - 1216. DOI:10.1109/18.850663.

3. Douik, A., Sorour, S., Al-Naffouri, T.Y., Alouini, M.-S. (2016) Instantly decodable network coding for real-time device-to-device communications. EURASIP Journal on Advances in Signal Processing, 1(2016), DOI: 10.1186/s13634-015-0293-z.

4. Douik, A., Sorour, S., Al-Naffouri, T.Y., Alouini, M.-S. (2017a) Decoding-Delay-Controlled Completion Time Reduction in Instantly Decodable Network Coding. IEEE Transactions on Vehicular Technology, 66 (3), 2756-2770. DOI: 10.1109/ TVT.2016.2585381.

5. Douik, A., Sorour, S., Al-Naffouri, T.Y., Alouini, M.-S. (2017b) Instantly Decodable Network Coding: From Centralized to Device-to-Device Communications. IEEE Communications Surveys \& Tutorials, 19 (2), 1201-1224. DOI: 10.1109/COMST.2017.2665587.

6. Douik, A., Sorour, S., Al-Naffouri, T.Y., Alouini, M.-S. (2017c) Rate Aware Instantly Decodable Network Codes. IEEE Transactions on Wireless Communications, 16 (2), 998-1011. DOI: 10.1109/TWC.2016.2635638.

7. Asareh, A., Fujii, T. (2013) Delay Reduction Scheme Based on Fountain Coding for Wireless Relay Communication Systems. In: IEEE $78^{\text {th }}$ Vehicular Technology Conference (VTC Fall), Sept. 2013, Las Vegas, NV, USA: IEEE, 1-6.

8. Rhoades, B. B., Conrad, J. M. (2017) A Survey of Alternate Methods and Implementations of an Intelligent Transportation System. In: IEEE Southeast Conference, April 2017, Charlotte, NC, USA: IEEE, 1-8.

9. Greco, C., Nemoianu, I.D., Cagnazzo, M., Pesquet-Popescu, B. (2016) Rate-distortion-optimized multi-view streaming in wireless environment using network coding. EURASIP Journal of Advanced Signal Processing, 17 (2016), DOI:10.1186/s13634-016-0308-4

10. MacKay, D. J. C. (2005) Fountain codes. IEEE Proceedings-Communications, 152 (6), 1062-1068. DOI: 10.1049/ip-com: 20050237.

11. Nguyen, D., Nguyen, T., Yang, X. (2007) Multimedia wireless transmission with network coding. In: Packet Video 2007, Nov 2007. Lausanne, Switzerland, Switzerland: IEEE, 326-335.

12. Nguyen, D., Nguyen, T. (2009) Network coding-based wireless media transmission using POMDP. In: $17^{\text {th }}$ International Packet Video Workshop, May 2009, Seattle, WA, USA: IEEE, 1-9. 
13. Drinea, E., Fragouli, C., Keller, L. (2009) Delay with network coding and feedback. In: IEEE International Symposium on Information Theory, July 2009, Seoul, South Korea: IEEE, 844-848.

14. Skevakis, E., Lambadaris, I. (2016) Optimal Control for Network Coding Broadcast. In: IEEE Global Communications Conference (GLOBECOM), December 2016, Washington, DC, USA: IEEE, 1-6.

15. Monteiro, F. A., Burr, A., Chatzigeorgiou, I., Hollanti, C., Krikidis, I., Seferoglu, H., Skachek, V. (2017) Special issue on Network Coding. EURASIP Journal on Advances in Signal Processing, 29. DOI: 10.1186/s13634-017-0463-2.

16. Qureshi, J. (2016) Random linear fountain code with improved decoding success probability. In: $22^{\text {nd }}$ Asia-Pacific Conference on Communications (APCC), Aug. 2016, Yogyakarta, Indonesia: IEEE. 6-11.

17. Sun, K., Zhang, H., Wu, D., Zhuang, H. (2016a) MPC-based Delay-Aware Fountain Codes for RealTime Video Communication. IEEE Internet of Things Journal, 5 (1), 415-424. DOI: 10.1109/JIOT.2016.2577520.

18. Sun, K., Zhang, H., Wu, D. (2016b) Delay-aware fountain codes for video streaming with optimal sampling strategy. arXiv eprint arXiv: 1605.03236.

19. Karim M.S., Sadeghi, P., Sorour, S. (2016) Instantly decodable network coding for real-time scalable video broadcast over wireless networks. EURASIP Journal of Advanced Signal Processing, 3 (2016). DOI: 10.1186/s13634-015-0299-6.

20. Hayajneh, K. F., Yousefi, S. (2015) Overlapped fountain coding for delay-constrained priority-based broadcast applications. IEEE Workshop on Information Theory (CWIT), St. John's, NL: IEEE. 79-82.

21. Hai, L., Wang, H., Wang, J. (2016) Instantly Decodable Network Coding for Multiple Unicast Retransmissions in Wireless Point-to-Multipoint Networks. IEEE Transactions on Vehicular Technology, 65 (8), 6232-6243. DOI: 10.1109/ TVT.2015.2472292.

22. Yu, M., Sadeghi, P., Aboutorab, N. (2015) Performance characterization and transmission schemes for instantly decodable network coding in wireless broadcast. EURASIP Journal of Advanced Signal Processing, 94 (2015), 1-17, DOI: 10.1186/s13634-015-0279-x.

23. Yu, M., Sadeghi, P., Sprintson, A. (2017) Feedback-Assisted Random Linear Network Coding in Wireless Broadcast. In: IEEE Globecom Workshops (GC Wkshps), Dec 2016, Washington, DC, USA: IEEE, 1-6.

24. Karim M.S., Douik, A., Sadeghi, P., Sorour, S. (2017) On Using Dual Interfaces With Network Coding for Delivery Delay Reduction. IEEE Transactions on Wireless Communications, 16 (6), 3981-3995. DOI: 10.1109/TWC.2017.2690436.

25. Gul, M.T., Ali, A., Singh, D.K., Imtinan, U., Raza, I., Hussain, S.A., Suh, D.Y., Lee, J.-W. (2016) Merge-and-forward: a cooperative multimedia transmissions protocol using RaptorQ codes. IET Communications, 10 (15), 1884-1895. DOI: 10.1049/iet-com.2016.0146.

26. Vineeth, N., Guruprasad H.S. (2014) The Influence of Network Coding on The Performance of Wireless Networks: A Survey. International Journal of Advanced Computer Technology, 3 (6), 884892. ISSN: 2320-0790.

27. Vineeth, N., Guruprasad H.S. (2015a) Delay Analysis of Network Coded Video Streams in VANETs. International Journal of Information Engineering and Electronic Business, 4, 16-23. DOI: 10.5815/ijieeb.2015.04.03.

28. Vineeth, N., Guruprasad H.S. (2015b) Performance analysis of network coded video streams in VANETs based on mobility models. In: IEEE International Advance Computing Conference (IACC), June 2015, Bangalore, India: IEEE, 170-175.

29. Vineeth, N., Guruprasad H.S. (2017a) The influence of the packet size on End to End Delay of video data coded with RaptorQ codes and Network Codes in Vehicular Adhoc Networks. ICTACT Journal on communication technology, 8 (3), 1582-1591. DOI: 10.21917/ ijct.2017.0233.

30. Vineeth, N., Guruprasad H.S. (2017b) A Suggestive Technique for the Reduction of End to End Delay during Data Dissemination in Vehicular Adhoc Networks. Journal of Engineering, Science and Management Education, 10 (2), 9. ISSN: 0976-0121.

31. Vineeth, N., Guruprasad H.S. (2018) Reduction of end-to-end delay using threaded RaptorQ codes in VANETs. International Journal of Vehicle Safety, 10 (2), 122-137. DOI: 10.1504/IJVS.2018.10015414.

32. Ambadi, N., Kumar, G.P., Rajan, B.S. (2017) Optimized Instantly Decodable Network Coding Protocols with Unequal Error Protection. In: IEEE Wireless Communications and Networking Conference (WCNC), March 2017, San Francisco, CA: IEEE, 1-6.

33. Sorour, S., Valaee, S. (2010) On Minimizing Multicast Completion Delay for Instantly Decodable Network Coding. In: International Conference on Computing, Networking and Communications (ICNC), May 2010, Cape Town, South Africa, 555-559. 
34. Sorour, S., Valaee, S. (2012) On densifying coding opportunities in instantly decodable network coding graphs. In: IEEE International Symposium on Information Theory Proceedings, July 2012, Cambridge, MA, USA: IEEE, 2456-2460.

35. Sorour, S., Valaee, S. (2013) Coding Opportunity Densification Strategies for Instantly Decodable Network Coding. IEEE Transactions on Communications, 61 (12), 5077-5089. DOI: 10.1109/TCOMM.2013.102313.110741.

36. Sorour, S., Valaee, S. (2015) Completion Delay Minimization for Instantly Decodable Network Codes. IEEE / ACM Transactions on Networking, 23 (5), 1553-1567. DOI:10.1109/TNET.2014.2338053.

37. Shokrollahi, A., Luby, M., Watson, M., Stockhammer, T. (2011) Raptorq forward error correction scheme for object delivery. Internet Engineering Task Force, Request for Comments: 6330.

38. Taghouti, M., Lucani, D. E., Pedersen, M. V., Bouallegue, A. (2016) Random linear network coding for streams with unequally sized packets: Overhead reduction without zero-padded scheme. In: International Conference on Telecommunications (ICT), May 2016, Thessaloniki, Greece: IEEE, 1-6.

39. Traskov, D., Medard, M., Sadeghi, P., Koetter, R. (2009) Joint Scheduling and Instantaneously Decodable Network Coding. In: IEEE Global Telecommunications Conference, Dec. 2009, Honolulu, HI: IEEE, 1-6.

40. Sui, Y., Wang, X., Wang, J., Wang, L., Hou, S. (2016) Deadline-aware cooperative data exchange with network coding. Computer Networks, 97, 88-97. DOI: 10.1016/ j.comnet.2016.01.003.

41. Heo, Y., Kim, H., Yun, J., Cho, Y., Cho, S., Lee, J. (2015) True realtime multimedia streaming system based on MMT. In: IEEE International Conference on Multimedia \& Expo Workshops (ICMEW), July 2015, Turin, Italy: IEEE, 1-3.

42. MacHardy, Z., Khan, A., Obana, K., Iwashina, S. (2018) V2X Access Technologies: Regulation, Research, and Remaining Challenges. IEEE Communications Surveys \& Tutorials, 20 (3), 18581877. DOI: 10.1109/ COMST.2018.2808444.

43. Zhang, H., Wen, Y., Huang, Q., Wu, D. (2016) File Transfer between Charging Stations Goes Infra structureless: A Code-Delay Tradeoff. IEEE Transactions on Vehicular Technology, 65 (10), 86828695. DOI: 10.1109/TVT.2015.2504496. 